\title{
IT-Controlling und IT-Produktivität
}

\author{
DOI 10.1007/s11576-009-0164-z
}

\section{Die Autoren}

Prof. Dr. Georg Rainer Hofmann

Hochschule Aschaffenburg

Würzburger Str. 45

63743 Aschaffenburg

Deutschland

hofmann@fh-aschaffenburg.de

\section{Prof. Dr. Ulrich Frank}

Lehrstuhl für Wirtschaftsinformatik

und Unternehmensmodellierung

Universität Duisburg-Essen

Campus Essen, Gebäude R09

Wirtschaftsinformatik

Universitätsstr. 9

45141 Essen

Deutschland

ulrich.frank@uni-due.de

This article is also available in English via http://www.springerlink.com and http://www.bise-journal.org Hofmann GR, Frank U (2009) IT Controlling and IT Productivity. Bus Inf Syst Eng. doi 10.1007/s12599-009-0049-0.
Informationstechnologie (IT) stellt keinen Selbstzweck dar. Sie dient dazu, die Effektivität und Effizienz von Prozessen und Entscheidungen in Organisationen zu fördern. IT leistet dazu i. d. R. einen offensichtlichen Beitrag. Dabei ist nicht allein an die Beschleunigung von Prozessen oder an die Verbesserung der Qualität von Entscheidungen zu denken. Daneben hat der innovative Einsatz von IT neue Geschäftsmodelle und Dienstleistungen ermöglicht, die einzigartige unternehmerische Erfolgsgeschichten beförderten. Diesen erheblichen Nutzenpotenzialen stehen allerdings auch beachtliche, durch den Einsatz von IT verursachte, Kosten und Risiken gegenüber. Diese sind u. a. auf die zum Teil erhebliche Komplexität von IT-Systemen, die Unsicherheiten über die zukünftige Entwicklung des relevanten IT-Marktes und die Unwägbarkeiten der Durchführung von IT-Projekten zurückzuführen. Aus wirtschaftlicher Perspektive ist dabei nicht zuletzt unbefriedigend, dass sich die Erfassung und Bewertung der mit der Realisierung und dem Betrieb von IT-Systemen verbundenen Kosten und Nutzen erheblichen Problemen gegenübersieht. Vor diesem Hintergrund ist es naheliegend, eine dedizierte organisatorische Funktion vorzusehen, die darauf zielt, die Wirtschaftlichkeit des Einsatzes von IT zu fördern, die wechselseitige Ausrichtung von Unternehmenszielen und IT-Zielen zu betonen und die Koordination von IT-Abteilung und Fachabteilungen zu unterstützen. Im deutschsprachigen Raum hat sich dafür der Begriff "IT-Controlling" eingebürgert, was insofern bemerkenswert ist, als es sich hier um eine offensichtlich englischsprachige Wortkonstruktion handelt, die im angelsächsischen Raum jedoch nicht gebräuchlich ist.

In den letzten Jahren haben die mit IT-Controlling assoziierten Aufgaben zunehmend an Bedeutung gewonnen. Hier ist an die weiter gestiegene Durchdringung von Organisationen mit IT zu denken, aber auch an die Probleme, die sich durch gewachsene, oft heterogene und unzureichend integrierte IT-Landschaften ergeben. Darüber hinaus agieren viele Branchen in einer zunehmend instabilen Umwelt, wodurch eine rasche und kostengünstige Anpassung der IT zu einem wesentlichen Wettbewerbsfaktor wird. Die damit verbundenen Entscheidungen über grundlegende Architekturkonzepte und Basistechnologien haben eine erhebliche wirtschaftliche Tragweite. Sie erfordern differenzierte Analysen der Kosten und Nutzeffekte der vorhandenen IT-Infrastruktur sowie möglicher Alternativen. Das in vielen Organisationen deutlich gestiegene Kostenbewusstsein hat zudem den Anspruch bestärkt, die Tätigkeit der IT-Abteilung in nachvollziehbarer Weise auf die Unterstützung des Kerngeschäfts auszurichten.

Die Praxis hat auf diese Entwicklung reagiert. In den letzten Jahren ist eine Reihe entsprechender Ansätze entstanden, von denen einige eine beachtliche Verbreitung erreicht haben. Sie zielen auf die Erhöhung der Transparenz und auf die Einführung klarer Richtlinien für die Erbringung von IT-Leistungen. Hier sind grundlegende Leitbilder wie "IT-Governance" und vor allem Rahmenwerke wie ITIL (IT Infrastructure Library) oder COBIT (Control Objectives for Information and related Technologies) zu nennen, die eine zielgerichtete Strukturierung und Koordination von IT-Leistungen unterstützen. Jenseits einer mitunter wenig seriösen Verwendung bietet das Konzept "Service" dabei die Chance, durch die Abstraktion auf IT-Services Komplexität zu reduzieren. Das Bemühen um Transparenz findet zudem Ausdruck in einer verstärkten Nachfrage nach Kennzahlensystemen, die eine verdichtete, entscheidungsorientierte Abbildung verschiedener Aspekte der Wirtschaftlichkeit von IT versprechen. Einige Anbieter von Software zur Unterstützung des IT-Managements haben diesen Trend erkannt. Sie zielen darauf, ihre Systeme durch eine Integration mit betriebswirtschaftlichen Anwendungssystemen aufzuwerten und ansprechende Visualisierungen für das Management zu bieten. 
Es ist unstrittig, dass IT-Controlling ein zentrales Thema der Wirtschaftsinformatik darstellt. Dies gilt in mehrfacher Hinsicht. So ist die Förderung der Wirtschaftlichkeit der Erstellung, Pflege und Nutzung von Informationssystemen ein wesentliches Forschungsziel der Disziplin. Gleichzeitig erfordert das IT-Controlling eine auch für die Wirtschaftsinformatik charakteristische Moderatorenfunktion zwischen Belangen der Betriebswirtschaftslehre und der Informatik. Nicht zuletzt gehört zu den zentralen Instrumenten des IT-Controllings ein dediziertes Informationssystem, dessen Gestaltung natürlich auch die Kernkompetenzen der Wirtschaftsinformatik betrifft. Vor diesem Hintergrund erscheint es überraschend, dass IT-Controlling in der jüngeren wissenschaftlichen Forschung nicht ein seiner Bedeutung entsprechender Stellenwert zukommt. Dies ist auch deshalb bemerkenswert, weil schon vor geraumer Zeit einige einschlägige Arbeiten, etwa im Rahmen des Informationsmanagement, vorgelegt wurden. Über mögliche Gründe dafür soll hier nicht spekulieren werden. Vielmehr ist festzustellen, dass die Zeit für eine Intensivierung der Forschung im Bereich des ITControllings günstig scheint. Das liegt zum einen an dem bereits erwähnten Unterstützungsbedarf in der Praxis. Zum anderen wurde der Methodenfundus der Wirtschaftsinformatik in den letzten Jahren um Instrumente erweitert, die grundsätzlich auch zur Unterstützung des IT-Controllings geeignet sind. Dadurch eröffnen sich Perspektiven auf zahlreiche reizvolle Forschungsthemen. Es ist deshalb erfreulich, dass IT-Controlling in jüngster Zeit eine zunehmende Aufmerksamkeit in der Forschung erfährt. Vor diesem Hintergrund ist die Motivation zur Gestaltung dieses Schwerpunkthefts zum Thema "IT-Controlling und IT-Produktivität" entstanden. Es zielt darauf, einen Überblick über den Stand der Forschung zu geben, aktuelle Forschungsansätze vorzustellen und zu weiterer Forschung in diesem Bereich anzuregen.

Der Beitrag von Stefan Strecker und Herbert Kargl untersucht, auch unter Rückgriff auf eine Rekonstruktion der Entwicklungsgeschichte, Integrationsdefizite des IT-Controllings im Verhältnis zum Methodenfundus der Wirtschaftsinformatik und zu Ansätzen in der Praxis. Reifegradmodelle für das IT-Management stellen ein wichtiges Instrument dar, weil sie die Positionierung der eigenen Organisation ermöglichen und Entwicklungsperspektiven aufzeigen. Der Beitrag von Jörg Becker, Ralf Knackstedt und Jens Pöppelbuß vergleicht einige Vorgehensweisen miteinander. Die auf diese Weise erlangten Erkenntnisse werden zu einem allgemein anwendbaren Vorgehensmodell generalisiert und konsolidiert. Der Beitrag bietet eine Anleitung für eine methodisch fundierte Entwicklung und Evaluation von Reifegradmodellen für das IT-Management. Aus dem benachbarten Ausland Liechtenstein stammt der Beitrag von Jan vom Brocke, Christian Sonnenberg und Alexander Simons, welcher das Konzept der Potenzialmodellierung einführt, das eine wertorientierte Betrachtung von Gestaltungsalternativen der IT ermöglicht. Zur Konkretisierung wird die Wertbeurteilung von serviceorientierten Architekturen (SOA) behandelt; der praktische Nutzen des Konzepts wird an einem Beispiel aufgezeigt. Eine Fallstudie aus dem Kontext der BMW Gruppe beschreiben Eva Peggy Sekatzek und Helmut Krcmar. Der Beitrag adressiert das Spannungsfeld, das sich durch die standardmäßige Nutzung von ERP-Systemen sowie die häufig erforderlichen Modifikationen ergibt. Der Beitrag beschreibt ein Kennzahlensystem zur Leistungsmessung von ERP-Systemen in diesem Spannungsfeld. Im Beitrag von Denis Royer und Martin Meints wird ein multidimensionaler Ansatz zur Gestaltungs- und Entscheidungsunterstützung vorgestellt, der sich an das bekannte Konzept der Balanced Scorecard anlehnt. Er unterstützt die differenzierte Evaluation der organisatorischen Implementierung von ITSystemen. In der Rubrik "State of the Art" gibt Andreas Gadatsch einen Überblick über die wichtigsten Begriffe des IT-Controllings, und stellt diverse IT-ControllingAnsätze der letzten Jahre näher dar. Ein für den Beitrag erarbeiteter IT-ControllingAnsatz beschreibt in einem dreistufigen Life-Cycle-Modell die zentralen Prozesse des IT-Controllings.

Wir danken den Autoren, auch denen, deren Einreichungen nicht angenommen wurden. Zudem gilt unser Dank den beteiligten Gutachtern, die die eingereichten Beiträge mit großer Sorgfalt differenziert bewertet haben. Ihnen, den Lesern, wünschen wir eine interessante und anregende Lektüre. 